\title{
Enhanced ability of information gathering may intensify disagreement among groups
}

\author{
Hiroki Sayama $\odot^{*}$ \\ Center for Collective Dynamics of Complex Systems, Binghamton University, Binghamton, New York 13902-6000, USA; \\ Max Planck Institute for the Physics of Complex Systems, 01187 Dresden, Germany; \\ and Waseda Innovation Lab, Waseda University, Shinjuku, Tokyo 169-8050, Japan
}

(Received 14 March 2020; accepted 15 June 2020; published 2 July 2020)

\begin{abstract}
Today's society faces widening disagreement and conflicts among constituents with incompatible views. Escalated views and opinions are seen not only in radical ideology or extremism but also in many other scenes of our everyday life. Here we show that widening disagreement among groups may be linked to the advancement of information communication technology by analyzing a mathematical model of population dynamics in a continuous opinion space. We adopted the interaction kernel approach to model enhancement of people's information-gathering ability and introduced a generalized nonlocal gradient as individuals' perception kernel. We found that the characteristic distance between population peaks becomes greater as the wider range of opinions becomes available to individuals or the more attention is attracted to opinions distant from theirs. These findings may provide a possible explanation for why disagreement is growing in today's increasingly interconnected society, without attributing its cause only to specific individuals or events.
\end{abstract}

DOI: $10.1103 /$ PhysRevE.102.012303

\section{INTRODUCTION}

Today's society faces many urgent critical challenges. One such challenge is addressing the widening disagreement and conflicts among different social constituent groups with incompatible views on politics, economies, international relationships, religions, cultures, lifestyle, and other aspects of our life.

Studies on this challenge often focus on how escalated views and opinions emerge in society [1]. Typical approaches in this area include detection of extremism in online media [2-4] and modeling contagious processes of extremism through social networks $[5,6]$. While highly relevant to and valuable for national security concerns, these approaches necessarily impose an asymmetric point of view to consider one side as the cause of the problem ("us" being normal versus "them" being abnormal), making it difficult to obtain a more system-oriented understanding of how such conflicts may arise and widen spontaneously at a global societal scale.

Escalated views and opinions are seen not only in radical ideology or extremism, but also in many other scenes of our everyday life (typically in a milder form), such as political conversations in social media $[7,8]$, health-care choices (e.g., the antivax movement) $[9,10]$, and dietary preferences $[11,12]$, to name a few. The widening disagreement among

\footnotetext{
*sayama@binghamton.edu; http://bingweb.binghamton.edu/ $\sim$ sayama/
}

Published by the American Physical Society under the terms of the Creative Commons Attribution 4.0 International license. Further distribution of this work must maintain attribution to the author(s) and the published article's title, journal citation, and DOI. Open access publication funded by the Max Planck Society. those who have escalated views is becoming more prevalent than before on a variety of subjects. Part of the cause is often suspected to be the recent advances of information communication technology (e.g., web media, social media, smart phones, and other forms of high-speed, high-volume, personalized communication) [9,13-15] that continuously increase information-gathering intensity and enhance users' ability to choose their preferred information sources (with some caveats [16]). In this view, widening disagreement may be modeled and understood as a spontaneous pattern formation process in which people's information-gathering ability plays a key role as the control parameter. The present study explores this view through mathematical modeling and analysis of opinion dynamics.

In this study, we combine the opinion dynamics with spatial models studied in mathematical biology. Specifically, we describe the dynamics of popularities of a wide range of opinions in partial differential equations (PDEs), inspired by models of diffusion and migration of biological organisms $[17,18]$. In particular, we adopt a model of autoaggregation [19-22] in which organisms aggregate together through a hill-climbing migration behavior on a terrain of signals. In our case, we consider people migrating in a space of opinions, and their migration is driven by the gradient of the opinion popularity itself.

We also propose a principled way to generalize a local gradient into a nonlocal perceived gradient based on the interaction kernel approach used in physics, applied mathematics, and mathematical biology. This allows us to model the enhancement of people's information-gathering ability, which would not be captured by simply using a conventional local gradient at a single point in the opinion space. It also allows for parametrization of two distinct aspects of nonlocal perception: the breadth of information gathering and the level of selective attention paid to distant opinions, the latter of which we hypothesize to play a particularly significant role in 
(a)

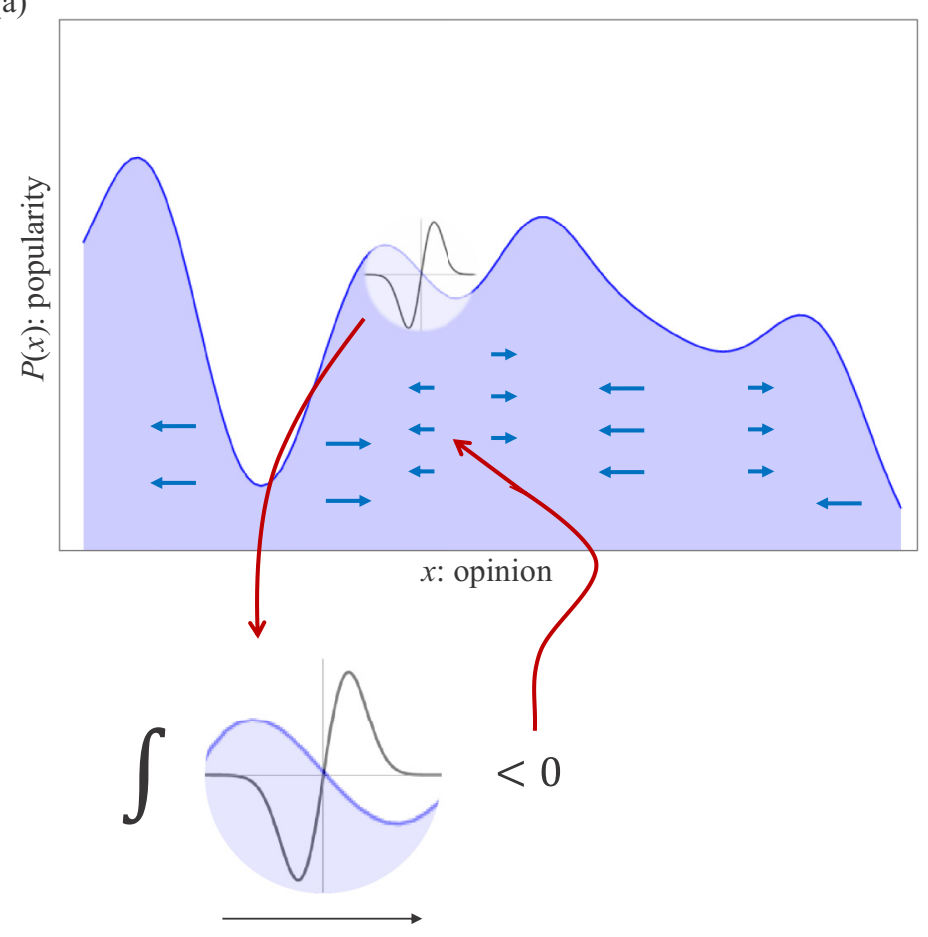

(b)

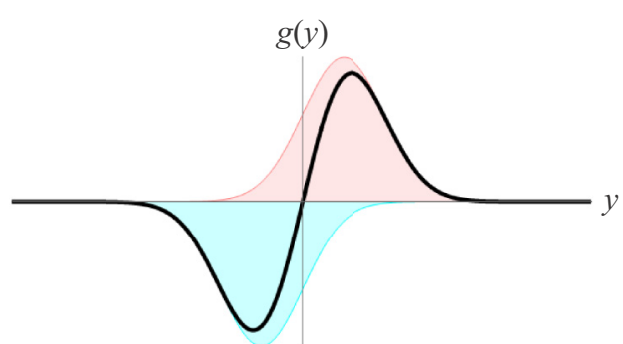

(c)

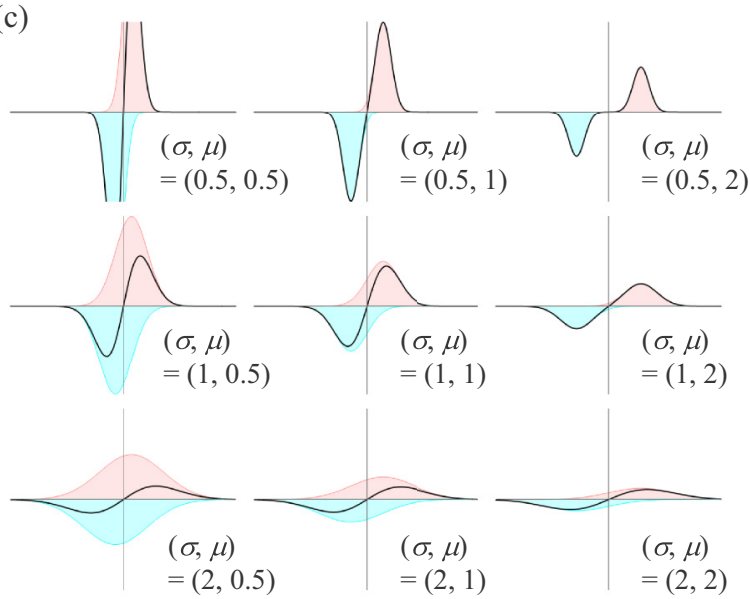

FIG. 1. Schematic overview of the mathematical model proposed in this study. (a) Popularities of opinions represented as distribution $P(x, t)$ on opinion $x . P(x, t)$ follows diffusion-migration dynamics described in Eq. (1), which may show, under typical parameter settings, aggregation behaviors as illustrated by small horizontal arrows in this figure. Directions of migrations are determined by a perceived gradient $G(P)$ defined as a cross-correlation between $P(x, t)$ and a perception kernel $g(y)$ at each location $x$ [Eq. (2), also depicted at the bottom of this panel]. (b) Structure of the perception kernel $g(y)$ [Eq. (3)]. The black solid curve shows the shape of $g(y)$, which is the sum of two Gaussian distributions with opposite signs (positive in pink and negative in cyan), one placed on the right and another on the left. (c) Variations of shapes of $g(y)$ as $\sigma$ and $\mu$ are varied. Having sharp peaks near the center in $g(y)$ (top left) makes $G(P)$ close to a conventional local derivative, corresponding to the case in which individuals' attention is limited only to opinions of similarly minded others. Having broader and/or distant peaks means enhanced information-gathering ability, covering a wider range of opinions and/or paying greater attention to distant opinions, respectively.

social opinion drift [23,24]. The model and the results of our analytical and numerical investigations are reported below.

\section{MODEL}

Our mathematical model represents the dynamics of popularities of opinions using a population distribution function $P(x, t)$ for opinion $x$ and time $t \geqslant 0$ [Fig. 1(a), top]. $P(x, t)$ is the number (in an arbitrary unit) of people whose current opinion is $x$ at time $t$. We assume the population never grows or decays, so the only changes possible in this model are due to diffusion and migration. Diffusion represents random fluctuations of people's opinions, while migration represents a directed, active change of people's opinions caused by social influence. More specifically, we adopt a widely used assumption [25-27] that people will more likely adopt opinions that are more supported by others. We also assume the homophily principle $[14,28,29]$ in people's information-gathering behavior, which implies that they perceive information only from a vicinity of their own opinion in the opinion space. The last two assumptions simplify the migration process into a simple hill-climbing behavior following the gradient of $P(x, t)$. Note that the opinion space modeled here is different from physical space within which individuals exchange opinions (e.g., social networks). Such social structure is not modeled explicitly in this study.

Both diffusion and migration can be modeled using the transport equation framework [18,22,30]. The model equation we use in this study to describe the dynamics of $P(x, t)$ is

$$
\frac{\partial P}{\partial t}=d \nabla^{2} P-c \nabla \cdot[P G(P)]
$$

where $d \nabla^{2} P$ is the diffusion term and $-c \nabla \cdot[P G(P)]$ is the migration term. $G(P)$ is the perceived gradient of popularity distribution, defined as

$$
\begin{aligned}
G(P) & =\int_{-\infty}^{\infty} P(x+y, t) g(y) d y, \\
g(y) & =\frac{1}{2 \mu} \frac{1}{\sqrt{2 \pi} \sigma}\left(e^{-\frac{1}{2}\left(\frac{y-\mu}{\sigma}\right)^{2}}-e^{-\frac{1}{2}\left(\frac{y+\mu}{\sigma}\right)^{2}}\right) .
\end{aligned}
$$

Equation (2) shows that the perceived gradient is defined as a cross-correlation between $P$ and a perception kernel $g$ given in Eq. (3) [Fig. 1(a), bottom], based on the interaction kernel approach commonly used in physics, applied mathematics, and mathematical biology $[21,30,31]$. The perception kernel 
$g$ describes how people assign weights (attentions) to nearby opinions' popularities when they assess the gradient. In this study, we define the perception kernel as a combination of two Gaussian distributions whose width is determined by $\sigma$ and whose means are separated by $2 \mu$ across the origin, one positive on the right-hand side and one negative on the left-hand side, to capture the difference of popularity levels between the two sides [Fig. 1(b)].

Note that $G(P)$ converges to a simple derivative $P^{\prime}(x)$ in the limit of $\mu \rightarrow 0^{+}$and $\sigma \rightarrow 0^{+}$, i.e., when $g$ is made of two Dirac's delta functions positioned right next to the origin with opposite signs (see Appendix A). This indicates that $G(P)$ can be considered a mathematically valid nonlocal generalization of a spatial derivative.

This generalization of the gradient enables us to explore different shapes of the perception kernel by varying $\sigma$ and $\mu$ [Fig. 1(c)] and to study their effects on opinion dynamics, which would not have been possible if only a local gradient were used. For example, increasing $\sigma$ [Fig. 1(c), bottom left] represents a situation in which people can gather information from a broader range of opinions. Meanwhile, increasing $\mu$ [Fig. 1(c), top right] represents a situation in which people tend to pay great attention particularly to distant, extreme opinions, which may correspond to sensationalism widely practiced in various media today.

\section{RESULTS}

\section{A. Stability analysis}

We first conduct a linear stability analysis of Eq. (1) to find the conditions under which homogeneous population distributions are unstable and thus heterogeneous patterns will form. We begin the analysis by replacing the spatiotemporal function $P(x, t)$ with a constant homogeneous population level $P_{h}$ plus a sinusoidal spatial perturbation with temporally changing small amplitude $\Delta P(t)$ [18], as follows:

$$
P(x, t) \rightarrow P_{h}+\Delta P(t) \sin (\omega x+\phi) .
$$

This replacement allows for linearization of Eq. (1) into the following nonspatial linear dynamical equation of $\Delta P$ (see Appendix B for details):

$$
\frac{d \Delta P}{d t}=\left(-d \omega^{2}+c \omega P_{h} \int_{-\infty}^{\infty} \sin (\omega y) g(y) d y\right) \Delta P .
$$

Therefore, with $Q(\omega)=\int_{-\infty}^{\infty} \frac{\sin (\omega y)}{\omega} g(y) d y$, if

$$
Q(\omega)>\frac{d}{c P_{h}}
$$

for $\omega>0$, then the homogeneous population distribution is unstable and a nonhomogeneous pattern (i.e., islands of popular opinions $=$ distinct groups) will form in the opinion space. This result already tells us that groups are more likely to form if (i) diffusion is weaker, (ii) active migration is stronger, and/or (iii) the population level is greater. These findings are consistent with results obtained for other similar autoaggregation models [17-20].

We also note that the range of $Q(\omega)$ is bounded to $[-1,1]$, and $Q(\omega)$ approaches its maximum 1 as $\omega \rightarrow 0$ regardless of the shape of the perception kernel (see Appendix C).
Therefore, in a sufficiently large opinion space, the lowfrequency perturbations always destabilize the homogeneous distribution eventually if and only if

$$
1>\frac{d}{c P_{h}} \quad \text { or } \quad c P_{h}>d
$$

which was also confirmed through numerical simulations (see Appendix D).

\section{B. Numerical study on the disagreement between groups}

Our main interest in this study is in the characteristic value of $\omega$ for instability. This is because the spatial period of perturbation, $L=2 \pi / \omega$, determines how far away the islands of opinions are separated from each other in the opinion space, which indicates the extent of disagreement between groups.

We numerically calculated the values of $Q(\omega)$ while varying $\sigma$ and $\mu$. Results are shown in Fig. 2, in which warmer colors indicate spatial frequencies $\omega$ that are more likely to destabilize the homogeneous population distribution (depending on the value of $\frac{d}{c P_{h}}$ as discussed above). These plots show that, the greater $\sigma$ and/or $\mu$ are, the more concentrated are the unstable perturbations on low-frequency regions, which correspond to greater distances between spontaneously formed groups.

Figure 3 shows actual numerical simulation results in a space-time plot for several values of $\sigma$ and $\mu$. The periodic boundary condition was used to avoid potential artifacts arising from cutoff spatial boundaries [32]. The population distribution initially remains more or less homogeneous for a certain period of time, but then distinct peaks (groups) quickly form. Once established, those groups become stable and remain unchanged for a substantially long period of time. Interestingly, the intervals between those peaks are longer for greater values of $\sigma$ and/or $\mu$, which can be interpreted as showing that the disagreement among those established groups becomes more intense as people's informationgathering ability is enhanced. It is also noticeable that the effects of $\sigma$ and $\mu$ are slightly different on the group formation process.

Figure 4 summarizes these results in a single plot of the characteristic inter-peak distance $L$ as a function of $\sigma$ and $\mu$ for $P_{h}=1, c=1$ and $d=0.2$. The surface plot shows a numerically obtained lower bound of $L$ based on the analysis [Eq. (6)]. Our analysis predicts that perturbations with a characteristic length below this surface would not grow. The peak distances obtained from numerical simulations (blue dots in Fig. 4) are all above this surface, which confirms that our analysis was valid. It is seen in the figure that the characteristic interpeak distance grows almost linearly with $\sigma$ and $\mu$, with a mild nonlinear interaction between them.

\section{Temporal change of information gathering ability}

This model allows us to test more dynamic scenarios in which people's information-gathering ability changes over time. Investigation of such hypothetical scenarios can provide us with valuable insight on potential interventions and possible societal responses to them. We test two hypothetical dynamic scenarios below.

The first scenario assumes that people's informationgathering ability gradually increases over time. This can be 
(a)

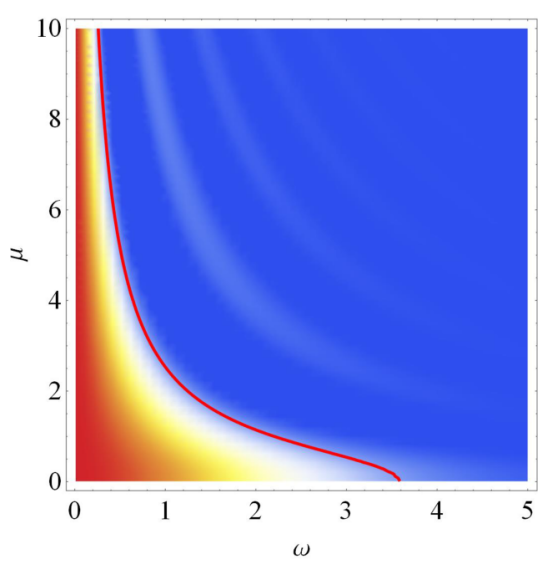

(b)

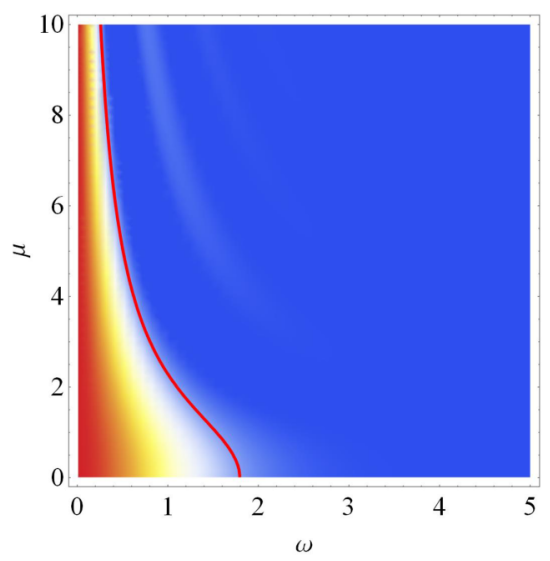

(c)

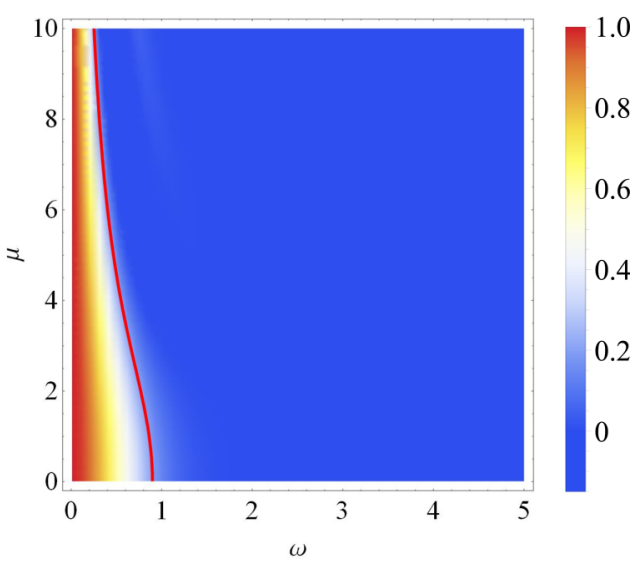

FIG. 2. Heatmaps showing the value of $Q(\omega)$ as a function of $\omega$ (horizontal axis), $\mu$ (vertical axis), and $\sigma$ [varied in three panels: (a) $\sigma=$ 0.5 , (b) $\sigma=1$, and (c) $\sigma=2$ ]. Red solid curves show contours that correspond to $Q(\omega)=\frac{d}{c P_{h}}=0.2$, the critical threshold under the parameter setting used for numerical simulations in this paper.

simulated by increasing values of $\sigma$ and $\mu$ in the course of a numerical simulation. This scenario imitates the gradual advancement of information communication technology, by which people gain access to a broader range of opinions (by greater $\sigma$ ) and pay greater attention to opinions distant from their own (by greater $\mu$ ). Figure 5(a) shows an illustrative example of this scenario, in which both $\sigma$ and $\mu$, initially set to 0.5 , begin to increase linearly with time at $t=20$, up to 5.0 by the end of the simulation at $t=200$. The smaller groups existing at the beginning gradually merge to form larger, more distant (more disagreeing) groups as $\sigma$ and $\mu$ increase. By the end of this particular simulation, more than a dozen of the initial small groups are integrated into just three large groups.

The second scenario models an attempt of external intervention to the population behavior observed in the first scenario. Specifically, we test what would happen if people's information-gathering ability were suppressed in the middle of the first scenario with an intention to dissolve the emerging larger groups. This scenario can be considered a simulation of government regulation or other forms of exogenous control, which can be simulated by lowering the values of $\sigma$ and $\mu$ after a certain period of group formation. Figure 5(b) shows an example of this scenario, which proceeds in the exact same way as in Fig. 5(a) for the first half, but then $\sigma$ and $\mu$ are suddenly reset to their initial value 0.5 at $t=100$ and remain constant thereafter. Interestingly, the large groups that are already established by the time of the intervention never become diffused, but to the contrary, they become more concentrated and more stable than before the intervention. This is because, unlike in other spatial biological/ecological models that have parameter-dependent characteristic scales of patterns [33-36], groups are generally stable in autoaggregation models like ours once they are established, regardless of parameter values of aggregation behavior. They may be absorbed into other groups or destroyed by sufficiently strong diffusion, but it is extremely difficult for them to disintegrate into smaller groups. This result implies that suppressing people's information gathering may not work as a means to dissolve those large groups with conflicting opinions, if they are already established.

\section{DISCUSSIONS AND CONCLUSIONS}

In this study, we proposed a PDE-based mathematical model of opinion dynamics in a continuous opinion space, and we studied its dynamics using both analytical and numerical means. Contributions of this work can be summarized in the following four points.

First, we presented an unconventional perspective to consider growing disagreement and conflicts in society the result of spontaneous pattern formation in an opinion space, in which the characteristic distance between population peaks represents how severe the disagreement is among distinct groups. This perspective allows us to understand intensifying disagreement as a system-level global property rather than a consequence caused by specific individuals or events to blame. Second, we proposed a generalized nonlocal spatial gradient and used it as a mathematical representation of the enhanced information-gathering ability of people. This enabled us to explore different shapes of perception kernels, and it also facilitated the linear stability analysis of the model. Third, we obtained several key analytical results on the general threshold between pattern-forming and non-patternforming regimes, as well as the effects of the parameters of information-gathering behavior on the distance between resulting groups, which were confirmed by numerical simulations. The result clearly showed that the distance among groups became greater as people's information-gathering ability was enhanced. Finally, we tested a few dynamic scenarios that produced relevant implications of increasing information communication technology for social dynamics and also some insight into the (lack of) effectiveness of external interventions.

Our results are generally in agreement with the now commonly made claim that the rapid development of the Internet, social media, smart phones, and other personal information communication technologies has contributed to the increase of societal conflicts and ideological escalation. Our informationgathering ability today is not comparable even to that of 20 years ago, and such a rapid change of our "perception kernel" may have already been producing emergent macroscopic 

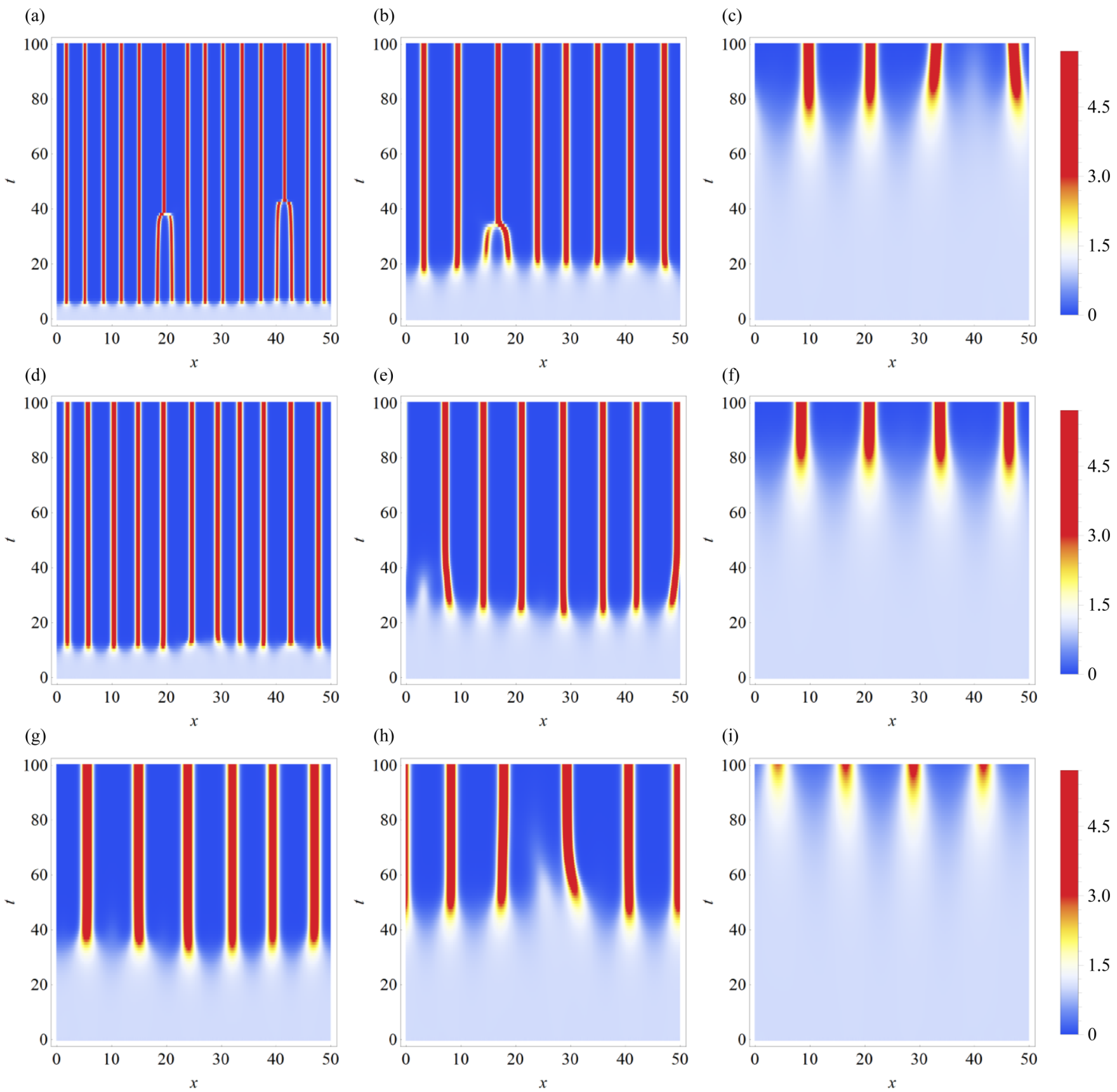

FIG. 3. Numerical simulation results of the population dynamics of the proposed model visualized in space ( $x$ : horizontal axis) and time $(t$ : vertical axis, going from bottom to top). Colors represent population density (blue $=0$, cold $=$ low, warm $=$ high, red $=3$ or above). $P_{h}=1$, $c=1$, and $d=0.2$. See Sec. V for details on the numerical integration. Results with several different values of $\sigma$ and $\mu$ are shown in this figure [left column (a,d,g): $\sigma=0.5$; center column (b,e,h): $\sigma=1.0$; right column (c,f,i): $\sigma=2.0$; top row (a,b,c): $\mu=0.5$; middle row (d,e,f): $\mu=1.0$; bottom row $(\mathrm{g}, \mathrm{h}, \mathrm{i}): \mu=2.0]$. The average distance $L$ between population peaks at $t=100$ was as follows: (a) $[(\sigma, \mu)=(0.5,0.5)]$ : $L=3.57143$; (b) $[(\sigma, \mu)=(1.0,0.5)]: L=6.25$; (c) $[(\sigma, \mu)=(2.0,0.5)]: L=12.5$; (d) $[(\sigma, \mu)=(0.5,1.0)]: L=4.54545 ;(\mathrm{e})[(\sigma, \mu)=$ $(1.0,1.0)]: L=7.14286$; (f) $[(\sigma, \mu)=(2.0,1.0)]: L=12.5 ;(\mathrm{g})[(\sigma, \mu)=(0.5,2.0)]: L=8.33333$; (h) $[(\sigma, \mu)=(1.0,2.0)]: L=10.0 ;(\mathrm{i})$ $[(\sigma, \mu)=(2.0,2.0)]: L=12.5$.

social patterns (such as those shown in Fig. 5) that go beyond any single individual's intention.

The results of the last scenario simulations illustrate challenging aspects of the observed opinion dynamics. As the perception kernel becomes wider and/or more focused on distant opinions, groups tend to merge hierarchically to eventually form a small number of large groups that are in significant disagreement from each other. Once they have formed, reducing the perception kernel would have no effect on their existence, but rather, it helps make those groups more crystallized. This leads us to questioning whether there are ways to remedy disagreement between those large groups and let them gracefully revert back to smaller groups with more distributed and more diversified opinions. 


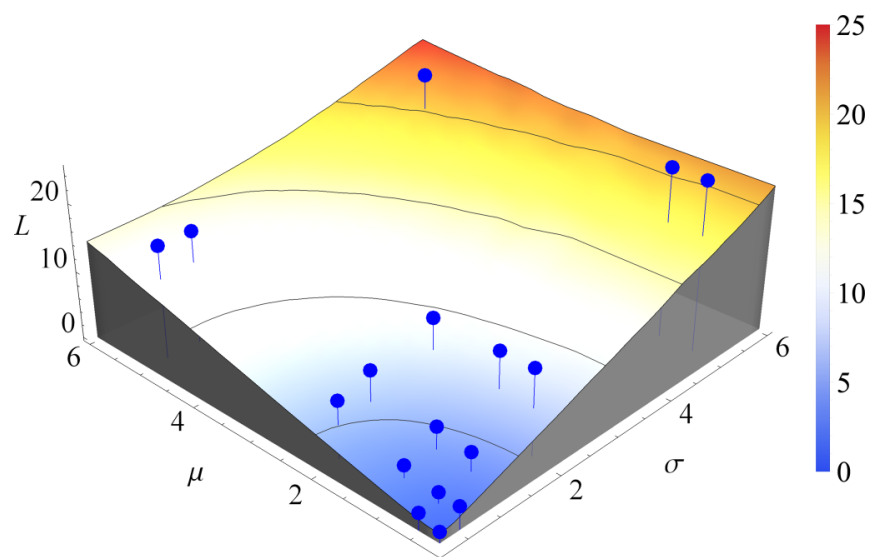

FIG. 4. Characteristic distance between population peaks $(L)$ visualized as a function of $\sigma$ and $\mu . P_{h}=1, c=1, d=0.2$. The surface plot shows a critical lower bound $L_{c}$ below which such perturbations would not grow. The lower bound $L_{c}$ was obtained as $L_{c}=\frac{2 \pi}{\omega_{c}}$, where $\omega_{c}$ is a numerically obtained critical value of $\omega$ that satisfies $Q(\omega)=\frac{d}{c P_{h}}$. The blue dots show actual peak distances measured in numerical simulations (e.g., Fig. 3), which are all above the surface. This confirms the validity of the analytical prediction that enhanced information-gathering ability (increased $\sigma$ and/or $\mu$ ) always results in a greater distance between population peaks.

Our model suggests that, at least mathematically, several different options exist for addressing this question. The first option is to increase the random diffusion rate $d$ or decrease the active migration rate $c$ so that Eq. (6) no longer holds. The second option is to reduce $\mu$ all the way to a negative value so that people seek originality rather than social conformity, changing the dynamics of the model from autoaggregation to autoavoidance. These two options are essentially suggesting altering people's behavior, but it is not obvious how one could achieve such global behavioral changes in reality (some welldesigned educational initiatives might help). The third option is to increase $\sigma$ and $\mu$ to extremely large values so that the boundaries of groups become more gradual and less defined [a sign of this phenomenon is seen near the end of the simulation in Fig. 5(a)]. The last option suggests to promote, rather than suppress, people's information gathering, but it would then bring another problem in that people's cognitive ability would be too limited to process the massive amount of information collected. None of these options is problem-free, but they may still suggest directions of potential solutions to be explored further.

We conclude this paper by pointing out several limitations of the study and identify future research questions. The model discussed in this study is still quite limited in both mathematical and practical aspects. Mathematically, we used only one form of the perception kernel to facilitate a parametrized representation of information-gathering behavior, but there should be many other functional forms that are plausible as a model of human information-gathering behaviors. For example, it was recently reported that exposure to distant opinions may have a repulsion effect [15], which was not considered in the present study but could be incorporated by revising the shape and sign of the perception kernel. Exploring different forms of the perception kernel and studying their effects on the resulting opinion dynamics would likely produce a more comprehensive understanding of this model. We also used only one boundary condition (periodic) in all of the numerical simulations presented, but the interactions of self-organizing patterns with nontrivial boundaries (i.e., the structure of possibilities in the idea space) are another area that warrants further systematic study.

In terms of practical aspects, it is certain that our model oversimplified the complexity of real human social dynamics. (a)

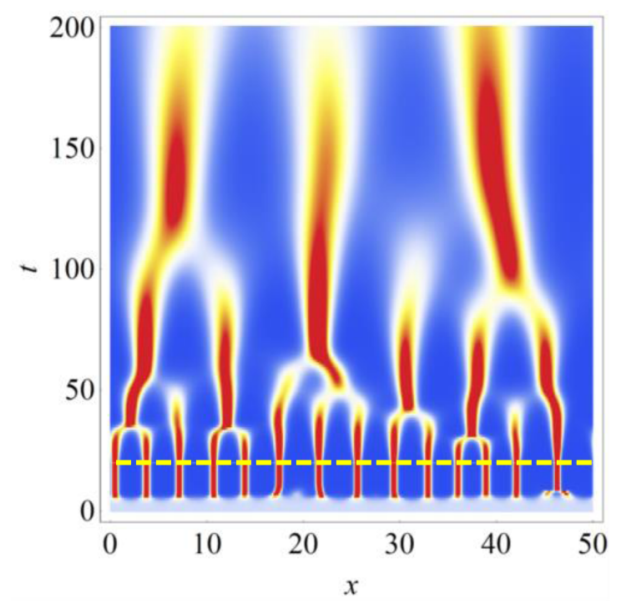

(b)

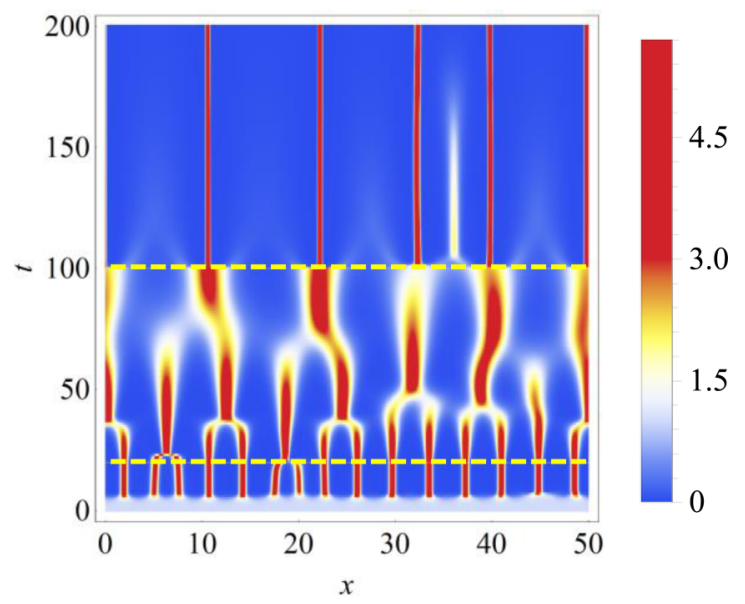

FIG. 5. Numerical simulation results of dynamic scenarios in which the values of $\sigma$ and $\mu$ are varied over time. The simulation method, initial condition, and meaning of colors are all the same as in Fig. 3. Simulations were conducted until $t=200$ in these plots. Yellow dashed lines represent key time points. (a) Initially $\sigma=\mu=0.5$, but after $t=20$ those parameters are linearly increased with time up to 5.0 by $t=200$. This scenario imitates the gradual advance of information communication technology. As $\sigma$ and $\mu$ increase, existing groups tend to merge to form larger, more distant (= disagreeing) groups over time. (b) Conditions are the same as in (a), except that $\sigma$ and $\mu$ are reset to their initial value 0.5 at $t=100$ and remain constant thereafter. This scenario imitates external intervention to reduce people's information-gathering ability, but it fails to diffuse the already established groups. 
We assumed only one-dimensional continuous opinion space, but opinions and ideas can be multidimensional. While we expect that the essential conclusions obtained from the stability analysis would still hold in multidimensional opinion space, details of implications would likely be influenced by the number of dimensions. In addition, this study did not explicitly consider the nontrivial social network structure of opinion exchange. The structure of society is implicit in this model, represented indirectly by the perception kernel (which allows individuals whose opinion states are close to each other to interact). In other words, the proposed model assumes that social connectivity is dynamically correlated with the proximity of opinions of individuals in the opinion space. Assumptions of a nontrivial social network structure with features such as heterogeneous degrees and communities are orthogonal to opinion dynamics. This can be incorporated into the model, but it is beyond the scope of the present study.

Furthermore, this study did not consider the behavioral diversity of individuals within the population at all. Such an assumption of homogeneous attributes shared among all components is still a common practice used in many theoretical studies of social dynamics, yet we have recently shown in a separate study that having even the simplest kind of individual diversity can greatly influence macroscopic behaviors of social systems [37]. Introducing behavioral diversity to the model may produce fundamentally different outcomes and implications. Finally, the proposed model has yet to be validated in comparison with quantitative real-world data. Equation (7) showed a unique dimensionless quantity $\frac{d}{c P_{h}}$ and its critical threshold 1 . This would allow at least for empirical testing of the global pattern formation condition using realworld data, regardless of specific choices of measurement units. Meanwhile, it might be difficult to obtain large-scale sociobehavioral data that could be directly used to test the effects of the perception kernel's shape, and therefore we anticipate the full model validation to be done through multiple hypothesis generation and testing.

\section{METHODS}

Numerical integration of the PDEs was conducted in a discretized space-time with spatial interval $\Delta x=0.05$ and temporal interval $\Delta t=0.001$ using a simple Euler-forward numerical integration method. The initial condition was a homogeneous population at $P_{h}=1$ everywhere in a spatial domain $[0,50]$, with small random perturbation (a random number sampled from a uniform distribution [-0.02, 0.02]) added to each discrete spatial location. The boundary condition was set to be periodic.

The numerical solver was implemented by the author in Julia 1.3.0, whose source code is available upon request.
Analysis and visualization of the simulation results were conducted using Wolfram Research Mathematica 12.0.0.

The data that support the findings of this study (source codes for numerical simulations, mathematical analysis, and visualization, as well as raw data for figures) are available from the author upon request.

\section{ACKNOWLEDGMENTS}

This work was supported by JSPS KAKENHI Grant No. 19K21571 and the Visitors Program of the Max Planck Institute for the Physics of Complex Systems.

\section{APPENDIX A: RELATIONSHIP BETWEEN LOCAL AND NONLOCAL GRADIENTS}

Here we show that the nonlocal gradient $G(P)$ introduced in this study converges to a simple local gradient (derivative) $P^{\prime}$ in the limit of $\mu \rightarrow 0^{+}$and $\sigma \rightarrow 0^{+}$, as follows:

$$
\begin{aligned}
\lim _{\mu, \sigma \rightarrow 0^{+}} G(P)= & \lim _{\mu \rightarrow 0^{+}} \frac{1}{2 \mu} \lim _{\sigma \rightarrow 0^{+}} \int_{-\infty}^{\infty} P(x+y) \\
& \times \frac{1}{\sqrt{2 \pi} \sigma}\left(e^{-\frac{1}{2}\left(\frac{y-\mu}{\sigma}\right)^{2}}-e^{-\frac{1}{2}\left(\frac{y+\mu}{\sigma}\right)^{2}}\right) d y \\
= & \lim _{\mu \rightarrow 0^{+}} \frac{P(x+\mu)-P(x-\mu)}{2 \mu} \\
= & P^{\prime}(x) .
\end{aligned}
$$

We also confirmed this convergence numerically (results not shown).

\section{APPENDIX B: DETAILS OF LINEAR STABILITY ANALYSIS}

We replace $P(x, t)$ in Eq. (1) with a homogeneous population level $P_{h}$ plus a sinusoidal spatial perturbation with temporally changing small amplitude $\Delta P(t)$, as follows:

$$
P(x, t) \rightarrow P_{h}+\Delta P(t) \sin (\omega x+\phi) .
$$

Then Eq. (1) is rewritten as follows:

$$
\begin{aligned}
\sin (\omega x+\phi) \frac{d \Delta P}{d t} \\
=-d \omega^{2} \sin (\omega x+\phi) \Delta P-c \frac{\partial}{\partial x}\left[\left[P_{h}+\Delta P \sin (\omega x+\phi)\right]\right. \\
\left.\quad \times \int_{-\infty}^{\infty}\left\{P_{h}+\Delta P \sin [\omega(x+y)+\phi]\right\} g(y) d y\right] .
\end{aligned}
$$

By ignoring the second-order term of $\Delta P$ and exploiting the fact that $g(y)$ is an odd function, this equation is linearly approximated as follows:

$$
\begin{aligned}
\sin (\omega x+\phi) \frac{d \Delta P}{d t} & \approx-d \omega^{2} \sin (\omega x+\phi) \Delta P-c P_{h} \frac{\partial}{\partial x} \int_{-\infty}^{\infty} \Delta P \sin [\omega(x+y)+\phi] g(y) d y \\
& =-d \omega^{2} \sin (\omega x+\phi) \Delta P-c \omega P_{h} \Delta P \int_{-\infty}^{\infty}[\cos (\omega x+\phi) \cos (\omega y)-\sin (\omega x+\phi) \sin (\omega y)] g(y) d y \\
& =-d \omega^{2} \sin (\omega x+\phi) \Delta P+c \omega P_{h} \sin (\omega x+\phi) \Delta P \int_{-\infty}^{\infty} \sin (\omega y) g(y) d y
\end{aligned}
$$


(a)

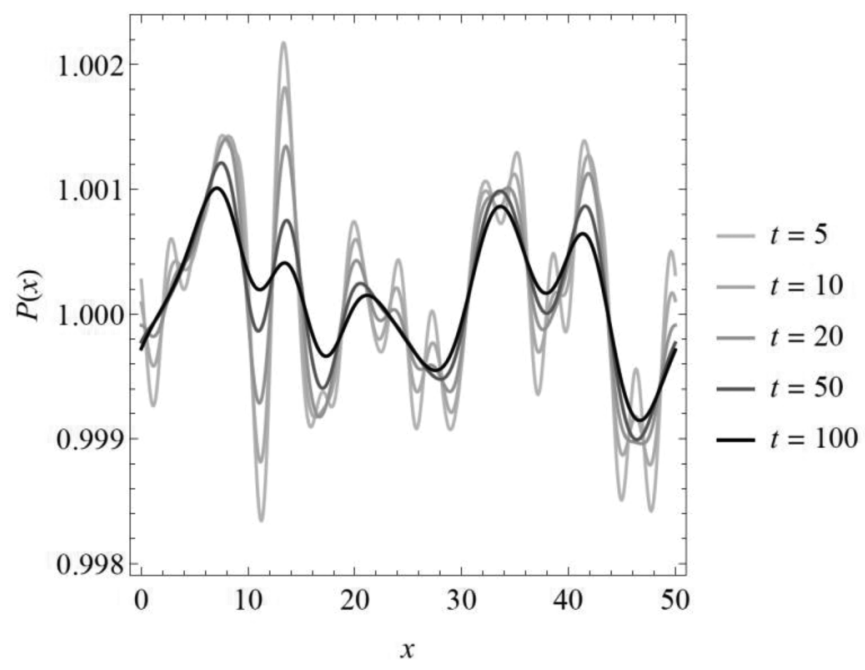

(b)

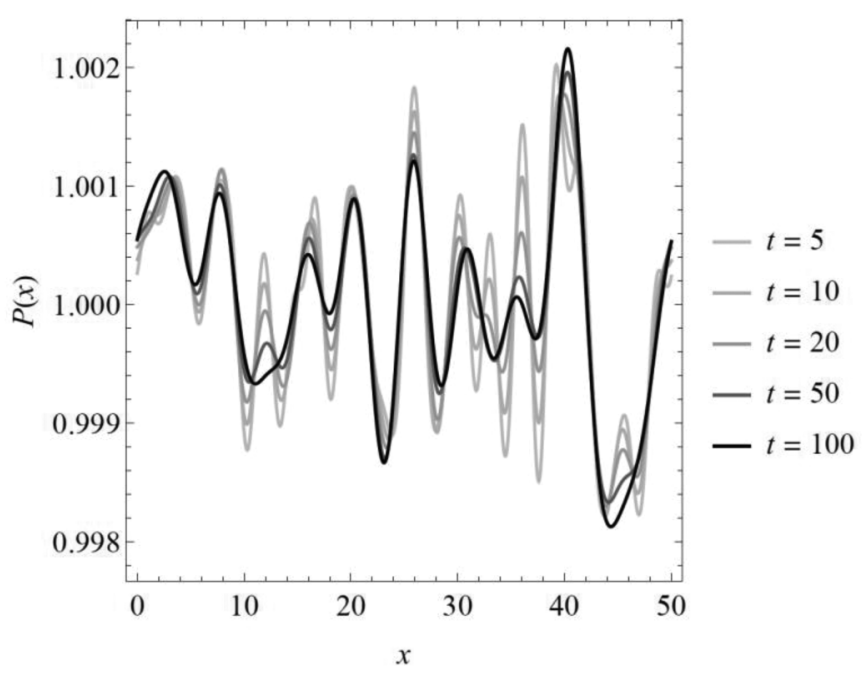

FIG. 6. Numerical simulation results with $c=P_{h}=1$ and $\sigma=\mu=0.1$. (a) Result with $d=1.01$, which is slightly above $c P_{h}=1$, therefore even the lowest-frequency perturbations gradually decay. (b) Result with $d=0.99$, which is slightly below $c P_{h}=1$, therefore lowest-frequency perturbations gradually grow and some peaks become more manifested over time. See Sec. V for details on the numerical integration. Note that the vertical axis is set on a small range to show the subtle difference between these two cases.

By dividing all terms by $\sin (\omega x+\phi)$ and collecting the coefficients of $\Delta P$ together, we obtain the following onedimensional linear dynamical equation of $\Delta P$ :

$$
\frac{d \Delta P}{d t}=\left(-d \omega^{2}+c \omega P_{h} \int_{-\infty}^{\infty} \sin (\omega y) g(y) d y\right) \Delta P .
$$

If the coefficient inside the parentheses above is positive, the small perturbation $\sin (\omega x+\phi)$ will grow, i.e., the homogeneous population distribution will be unstable, and nonhomogeneous patterns (distinct groups) will form.

With $Q(\omega)=\int_{-\infty}^{\infty} \frac{\sin (\omega y)}{\omega} g(y) d y$, this condition for pattern formation is summarized as

$$
Q(\omega)>\frac{d}{c P_{h}}
$$

for $\omega>0$, as described in the main text.

\section{APPENDIX C: PROPERTIES OF $Q(\omega)$}

We note that $Q(\omega)$ is, by itself, the generalized nonlocal gradient of $\sin (\omega x) / \omega$ around $x=0$. This indicates that the range of $Q(\omega)$ is bounded by the range of the gradients of the original function $\sin (\omega x) / \omega$, which is $\cos (\omega x)$, hence $Q(\omega) \in$ $[-1,1]$.

Moreover, we show that $Q(\omega)$ approaches its maximum 1 regardless of the shape of $g(y)$ in the limit of $\omega \rightarrow 0$, as follows:

$$
\begin{aligned}
\lim _{\omega \rightarrow 0} Q(\omega) & =\lim _{\omega \rightarrow 0} \int_{-\infty}^{\infty} \frac{\sin (\omega y)}{\omega} g(y) d y \\
& =\lim _{\omega \rightarrow 0} \int_{-\infty}^{\infty} y g(y) d y \\
& =1 .
\end{aligned}
$$

\section{APPENDIX D: NUMERICAL SIMULATIONS DEMONSTRATING THE $c P_{h}>d$ INSTABILITY CONDITION}

Our analysis shows that, if the opinion space is sufficiently large, the low-frequency perturbations always destabilize the homogeneous population distribution if and only if $c P_{h}>d$. This prediction can be confirmed through numerical simulations. Illustrative cases are shown in Fig. 6.
[1] Y.-Li. Chuang and M. R. D’Orsogna. Mathematical models of radicalization and terrorism, arXiv:1903.08485.

[2] E. Ferrara, W.-Q. Wang, O. Varol, A. Flammini, and A. Galstyan, Predicting online extremism, content adopters, and interaction reciprocity, in International Conference on Social Informatics (Springer, Cham, 2016), pp. 22-39.

[3] A. Badawy and E. Ferrara, The rise of jihadist propaganda on social networks, J. Comput. Social Sci. 1, 453 (2018).

[4] P. D. Manrique, M. Zheng, Z. Cao, E. M. Restrepo, and N. F. Johnson, Generalized Gelation Theory Describes Onset of Online Extremist Support, Phys. Rev. Lett. 121, 048301 (2018).

[5] J. M. Berger, The metronome of apocalyptic time: Social media as carrier wave for millenarian contagion, Perspect. Terrorism 9, 61 (2015).

[6] E. Ferrara, Contagion dynamics of extremist propaganda in social networks, Inf. Sci. 418, 1 (2017).

[7] M. D. Conover, J. Ratkiewicz, M. Francisco, B. Gonçalves, F. Menczer, and A. Flammini, Political polarization on Twitter, in Fifth International AAAI Conference on Weblogs and 
Social Media (AAAI Press, Menlo Park, California, 2011), pp. 89-96.

[8] A. J. Morales, J. Borondo, J. C. Losada, and R. M. Benito, Measuring political polarization: Twitter shows the two sides of Venezuela, Chaos 25, 033114 (2015).

[9] A. Kata, Anti-vaccine activists, Web 2.0, and the postmodern paradigm - an overview of tactics and tropes used online by the anti-vaccination movement, Vaccine 30, 3778 (2012).

[10] N. F. Johnson, N. Velasquez, N. J. Restrepo, R. Leahy, N. Gabriel, S. Wuchty, and D. Broniatowski, Health wars and beyond: The rapidly expanding and efficient network insurgency interlinking local and global online crowds of distrust, arXiv:1910.02103.

[11] M. Cole and K. Morgan, Vegaphobia: Derogatory discourses of veganism and the reproduction of speciesism in UK national newspapers, Br. J. Sociol. 62, 134 (2011).

[12] N. R. Reilly, The gluten-free diet: Recognizing fact, fiction, and fad, J. Pediatr. 175, 206 (2016).

[13] M. Prior, Media and political polarization, Ann. Rev. Polit. Sci. 16, 101 (2013).

[14] E. Bakshy, S. Messing, and L. A. Adamic, Exposure to ideologically diverse news and opinion on Facebook, Science 348, 1130 (2015).

[15] C. A. Bail, L. P. Argyle, T. W. Brown, J. P. Bumpus, H. Chen, M. B. Fallin Hunzaker, J. Lee, M. Mann, F. Merhout, and A. Volfovsky, Exposure to opposing views on social media can increase political polarization, Proc. Natl. Acad. Sci. (USA) 115, 9216 (2018).

[16] L. Boxell, M. Gentzkow, and J. M. Shapiro, Greater Internet use is not associated with faster growth in political polarization among US demographic groups, Proc. Natl. Acad. Sci. (USA) 114, 10612 (2017).

[17] L. Edelstein-Keshet, Mathematical Models in Biology (SIAM, Philadelphia, 2005).

[18] H. Sayama, Introduction to the Modeling and Analysis of Complex Systems (Open SUNY Textbooks, Geneseo, New York, 2015).

[19] E. F. Keller and L. A. Segel, Initiation of slime mold aggregation viewed as an instability, J. Theor. Biol. 26, 399 (1970).

[20] D. Horstmann, From 1970 until present: The Keller-Segel model in chemotaxis and its consequences I, Jahresbericht der Deutschen Mathematiker-Vereinigung 105, 103 (2003); From 1970 until present: The Keller-Segel model in chemotaxis and its consequences II, 106, 51 (2004).

[21] S. Boi, V. Capasso, and D. Morale, Modeling the aggregative behavior of ants of the species Polyergus rufescens, Nonlin. Anal. 1, 163 (2000).

[22] T. Hillen and K. J. Painter, A user's guide to PDE models for chemotaxis, J. Math. Biol. 58, 183 (2009).

[23] H. Sayama and R. Sinatra, Social diffusion and global drift on networks, Phys. Rev. E 91, 032809 (2015).
[24] H. Sayama, Going extreme without leaders, https: //medium.com/@hsayama/going-extreme-without-leaderse2f1ea0ba520, 2016. Accessed: 2020-01-06.

[25] M. H. DeGroot, Reaching a consensus, J. Am. Stat. Assoc. 69, 118 (1974).

[26] N. E. Friedkin and E. C. Johnsen, Social influence and opinions, J. Math. Sociol. 15, 193 (1990).

[27] S. Banisch and E. Olbrich, Opinion polarization by learning from social feedback, J. Math. Sociol. 43, 76 (2019).

[28] M. McPherson, L. Smith-Lovin, and J. M. Cook, Birds of a feather: Homophily in social networks, Annu. Rev. Sociol. 27, 415 (2001).

[29] G. Kossinets and D. J. Watts, Origins of homophily in an evolving social network, Am. J. Sociol. 115, 405 (2009).

[30] M. Di Francesco and S. Fagioli, Measure solutions for nonlocal interaction PDEs with two species, Nonlinearity 26, 2777 (2013).

[31] A. Mogilner and L. Edelstein-Keshet, A non-local model for a swarm, J. Math. Biol. 38, 534 (1999).

[32] Periodic boundary condition is chosen here only for numerical purposes. It would not be deemed a good assumption if this model were about polarization between two extremes. However, this is not a critical issue because the objective of this study is to characterize not such polarization but disagreement between opinionated groups, and also even the concept of polarization itself can be much more nuanced than simple binary division [38,39].

[33] A. M. Turing, The chemical basis of morphogenesis, Philos. Trans. R. Soc. London, Ser. B 237, 37 (1952).

[34] J. E. Pearson, Complex patterns in a simple system, Science 261, 189 (1993).

[35] H. Sayama, M. A. M. de Aguiar, Y. Bar-Yam, and M. Baranger, Spontaneous pattern formation and genetic invasion in locally mating and competing populations, Phys. Rev. E 65, 051919 (2002).

[36] S. Kondo and T. Miura, Reaction-diffusion model as a framework for understanding biological pattern formation, Science 329, 1616 (2010).

[37] H. Sayama and J. Yamanoi. Beyond social fragmentation: Coexistence of cultural diversity and structural connectivity is possible with social constituent diversity, in Proceedings of NetSci$X$ 2020: Sixth International Winter School and Conference on Network Science (Springer, Cham, 2020), pp. 171-181.

[38] A. Bramson, P. Grim, D. J. Singer, S. Fisher, W. Berger, G. Sack, and C. Flocken, Disambiguation of social polarization concepts and measures, J. Math. Sociol. 40, 80 (2016).

[39] A. Bramson, P. Grim, D. J. Singer, W. J. Berger, G. Sack, S. Fisher, C. Flocken, and B. Holman, Understanding polarization: Meanings, measures, and model evaluation, Philos. Sci. 84, 115 (2017). 